Estudios Públicos 165 (2022), I29-I 37

DOI: https://doi.org/|0.38|78/07/83089/|6022 I I002

Artículo

\title{
Una teoría de la justicia y de la familia. Las críticas feministas a Teoría de la justicia
}

\author{
Nicole Darat Guerra \\ Universidad Adolfo Ibáñez, Chile
}

\begin{abstract}
RESUMEN: El objetivo de este texto es dar cuenta de parte de la recepción feminista de Teoría de la justicia (TJ), principalmente de la reacción de las teóricas feministas a las ideas sobre la familia y las relaciones entre los sexos en TJ. Para ello procederé de la siguiente forma: comenzaré por (1) dar cuenta del estado del debate feminista en 1971, cuando apareció TJ; luego (2) haré una breve mención a las principales críticas que recibió $\mathrm{TJ}$; (3) a continuación me detendré puntualmente en la crítica al concepto de familia, por ser este uno de los aspectos más relevantes para la crítica feminista; (4) finalmente sugeriré en pocas líneas por qué el liberalismo político provee una base insuficiente para repensar la familia en clave feminista.
\end{abstract}

Palabras Clave: Rawls, crítica feminista, familia, posición original, estructura básica

RECIBIDO: octubre 2021 / ACEPTADO: diciembre 2021

\section{A Theory of Justice and of the Family. Feminist Critiques to A Theory of Justice}

ABSTRACT: The objective of this paper is to give an account of part of the feminist reception of $A$ Theory of Justice (TJ) mainly of the feminist thinkers' reaction to the ideas of family and gender relations in TJ. In order to do that I will proceed as follows, I will begin by summarizing the state of the feminist debate in 1971, when TJ was first published, (2) I will make a short mention to the main criticisms TJ received, (3) I

Nicole Darat Guerra es Doctora en Filosofía por la Universidad de Valladolid, España. Es Profesora Asistente de la Facultad de Artes Liberales, Universidad Adolfo Ibáñez, Viña del Mar, Chile. Dirección: Padre Hurtado 750, Viña del Mar, Chile, CP 2520000. Email: nicole.darat@ uai.cl. 
will focus specially in the critique of the concept of family, being this one of the most relevant for feminist criticism, (4) I will suggest in a few lines why political liberalism provides an inadequate basis to rethink family from a feminist perspective.

KEYWORDS: Rawls, feminist criticism, family, original position, basic structure

ReCEIVED: October / Accepted: December

\section{El contexto: la segunda ola feminista}

$E^{\text {na }}$ ntre 1970 y 1971 vieron la luz tres clásicos del llamado feminismo de la segunda ola: Sexual Politics de Kate Millet; Sisterhood Is Powefull, antología del feminismo radical compilada por Robin Morgan, y Dialectics of Sex de Shulamith Firestone.

La primera ola feminista se concentró en la consecución de los derechos formales que garantizaran la igualdad entre mujeres y varones, principalmente en el acceso a la educación, al voto y al mercado de trabajo. No obstante, una vez dichos logros fueron alcanzados, la posición social de las mujeres continuó siendo inferior a la de los varones. Es por ello que la segunda ola feminista se planteó la cuestión de las opresiones de género como un fenómeno diseminado en la sociedad, que excedía las instituciones políticas. Los roles de género y la división sexual del trabajo en la familia son los principales blancos de la segunda ola feminista, que comienza a poner en circulación el concepto de 'patriarcado' para entender la opresión de las mujeres en tanto que mujeres, independientemente de otras variables. Por ende, las primeras críticas feministas que recibió Teoría de la justicia (TJ) (2005) tuvieron como blanco la conceptualización de la familia y el lugar que ella ocupa en la obra.

\section{Las críticas feministas a TJ. Una revisión general}

Las críticas feministas a TJ se han centrado en cuatro puntos. Primero, en la noción de estructura básica y en el confuso lugar de la familia a lo largo de toda la obra, incluido su cuestionamiento a propósito del principio de diferencia y su importancia en la teoría del desarrollo moral. Segundo, en el lugar del género en relación con las diferencias arbitrarias que deberían quedar o no tras el velo de ignorancia. Tercero, respecto de 
la representación de las partes en la posición original como 'cabezas de familia'. En cuarto lugar destaca una crítica más general sobre el enfoque mismo de la teoría en la redistribución de recursos, que desatiende otras formas de injusticia en tanto que falta de reconocimiento.

Por las razones que expliqué en el primer apartado, me centraré en los puntos 1 y 3, y en menor medida en 2. En cuanto al punto 4, Rawls dio respuestas más satisfactorias en trabajos posteriores.

\title{
3. ¿Qué dice Rawls sobre la familia en TJ?
}

\section{Como parte de la estructura básica}

\begin{abstract}
Para nosotros, el objeto primario de la justicia es la estructura básica de la sociedad o, más exactamente, el modo en que las grandes instituciones sociales distribuyen los derechos y deberes fundamentales y determinan la división de las ventajas provenientes de la cooperación social. Por grandes instituciones entiendo la constitución política y las principales disposiciones económicas y sociales. Así, la protección jurídica de la libertad de pensamiento y de conciencia, la competencia mercantil, la propiedad privada de los medios de producción y la familia monógama son ejemplos de las grandes instituciones sociales. (Rawls 2005, § 2)
\end{abstract}

En una primera presentación, la familia es considerada dentro de este tipo de instituciones, es decir, se la considera como parte de aqueIlas instituciones que distribuyen derechos y deberes, y que, por consiguiente, afectan la vida de los ciudadanos. Pero la posición de Rawls sobre este asunto no es consistente a lo largo del texto, tal como veremos más adelante.

\section{La visión de las partes en la posición original (PO) como 'cabezas de familia'}

Esta visión le permite a Rawls hacer ciertas suposiciones motivacionales que harían plausible el principio de ahorros justos, de una generación a otra. El suponer que las partes en la PO son 'cabezas de familia' apuntala el supuesto de que los individuos no se preocuparán únicamente por aquello que afectará a su propia expectativa de vida posible, sino que deberán preocuparse por, al menos, la generación inmediatamente posterior. En TJ se referirá a las partes unas veces como 'cabezas de familia' y otras como 'líneas genéticas'. Rawls usa estos términos de forma inter- 
cambiable y el que así lo haga da cuenta de que tras la caracterización aparentemente neutra de la posición original, opera ya un supuesto sobre, por un lado, la homogeneidad de los intereses al interior de la familia y, por otro, de que quien puede representarlos es muy probablemente un varón.

Una de las críticas de Rawls al utilitarismo es que no se toma en serio la distinción entre las personas, pero cuando él concibe a las partes en la PO como cabezas de familia ¿se toma realmente en serio esta distinción?, ¿por qué esta no es una preocupación cuando se trata de las familias? Rawls no distingue a los individuos como sujetos de demandas diferenciadas dentro de la familia, pues concibe a la misma fundamentalmente como una unidad de amor. ¿Pero por qué la considera parte de la estructura básica?

Rawls es bastante austero en sus asunciones motivacionales, pero no tiene problemas en asumir que las partes representan una 'línea continua de demandas'.

\section{La familia en los principios de justicia}

Cuando explica las diferentes interpretaciones del segundo principio, al hacer referencia a la interpretación liberal, Rawls afirma:

Más aún, el principio de la igualdad de oportunidades solo puede realizarse imperfectamente, al menos mientras exista en alguna forma la institución de la familia. El grado en que se desarrollen y fructifiquen las capacidades naturales se ve afectado por todo tipo de condiciones sociales y actitudes de clase. Incluso la buena disposición para hacer un esfuerzo, para intentarlo, y por tanto ser merecedor del éxito en el sentido ordinario, depende de la felicidad en la familia y de las circunstancias sociales. (Rawls 2005, § 74)

Sobre este punto, Rawls señala que la interpretación liberal del segundo principio como igualdad justa de oportunidades, en tanto que iguales oportunidades para quienes tienen iguales talentos, sigue dejando demasiado a la fortuna, en el sentido de que los talentos son producto de la suerte y son arbitrarios desde un punto de vista moral. Mientras exista la familia, esta desigualdad seguirá reproduciéndose, pues nacer en el seno de una familia u otra, ser nutrido en una familia u otra, es una cuestión que está fuera de nuestro ámbito de decisión. Sin embargo, todavía hablando del principio de diferencia, Rawls hará referencia a la fa- 
milia como una analogía a partir de la cual podemos entender el tipo de fraternidad que pone en acción dicho principio, sin volver sobre el punto anterior, y a partir del cual explicar en qué sentido ambas visiones de la familia son compatibles. El autor declara que, "[e]n general, los miembros de una familia no desean beneficiarse a menos que puedan hacerlo de manera que promuevan el interés del resto" (Rawls 2005, § 18).

\section{La familia y el desarrollo moral}

Más adelante, cuando habla del desarrollo moral, Rawls vuelve a referirse a la familia como parte de la estructura básica, aunque ahora lo matiza afirmando que esta pertenece 'de alguna forma' a la estructura básica. ¿De qué forma?

El punto de partida del desarrollo moral, del cual depende el desarrollo del sentido de la justicia, es el amor de la familia hacia los hijos, y Rawls necesita asumir dicho amor como un hecho y a la familia como una institución relativamente aproblemática, lo que reafirma con el enunciado "dado que las instituciones de la familia son justas" (Rawls $2005, \S 75)$. ¿En qué sentido, entonces, son parte de la estructura básica si asumimos que son justas y, por ende, someterlas a los principios sería irrelevante?

El parágrafo 77 es referido como el lugar donde Rawls se hará cargo de la familia como reproductora de la injusticia entre los individuos de diferentes familias. Es ahí donde él lanza la provocadora pregunta de si acaso debería abolirse la familia para lograr una sociedad igualitaria. Para Rawls, la superación de la interpretación liberal por la interpretación democrática, que apela al principio de la diferencia, neutraliza de forma significativa la influencia de las familias en las perspectivas futuras de los individuos, al punto de que le resta urgencia a la respuesta a esa pregunta. La urgencia de esta pregunta quedaría desactivada por el principio de la diferencia en cuanto a las desigualdades económicas que la familia puede producir. No obstante, en cuanto a las desigualdades de género al interior de la familia, la sola existencia de la 'familia monógama', tal como Rawls la entiende en TJ, depende de una particular división sexual del trabajo, y por ende reproduce desigualdades de género, permaneciendo la desigualdad.

La familia tiene entonces un desarrollo complejo, dual si se quiere, pero esta dualidad no se explicita entre la familia ideal y la familia con- 
creta; al menos, esta distinción no recibe un tratamiento sistemático en TJ. Considerando la extensión del libro, creo que podemos atribuir correctamente un desinterés en Rawls por abordar la familia en su complejidad.

¿Cómo han reaccionado las críticas feministas a esta inconsistencia en TJ?

Haré referencia a tres críticas que se han hecho cargo de estos puntos. La primera, de Jane English en 1977, fue la primera respuesta feminista a TJ y se centra en la concepción de las partes como 'cabezas de familia'. La segunda, de Veronique Munoz-Darde (1998), retoma la crítica de English, pero profundizando en la familia como reproductora de desigualdades. La última, quizás la crítica feminista más influyente de TJ, es la de Susan Moller Okin, manifiesta en Justice, Gender and the Family (2007).

La crítica de English se centra en el supuesto de que las partes en la posición original son 'cabezas de familia' y critica a Rawls por abandonar el supuesto de que son individuos a secas. Entender una familia como una unidad implica borrar los conflictos en la distribución de bienes y cargas de la misma.

La idea de considerar la familia como un todo conduce a una lectura utilitarista que traiciona las propias intenciones de Rawls. Si la familia es un todo, el principio de ahorro justo podría contemplar formas de división del trabajo que son sexistas (por ejemplo, es más eficiente que el cuidado de los recién nacidos sea hecho por las mujeres, pues son quienes dan a luz; habría una cierta continuidad entre el amamantamiento y el resto de los cuidados, que justificaría esta división del trabajo y una consiguiente educación sexista). Pero esta lectura parece más apegada al principio de eficiencia que al principio de diferencia, que exigiría que cualquier distribución mejorara la situación de los peor situados. English (1977) propone otra forma de dirimir el conflicto interno de una familia, más acorde con los planteamientos de Rawls: hacer una 'miniconvención' al interior de la familia; poner a los miembros de la familia tras el velo de ignorancia en un 'velo tras el velo'. El resultado sería que ellos no aceptarían la idea del ahorro entre generaciones, pues si son racionales y autointeresados, los miembros de la familia no elegirían sacrificarse para mejorar el bienestar de la familia como un todo (English 1977, 95). 
English (1977) procede a criticar el principio de ahorros justos en sí mismo. ¿Qué es lo que se debe ahorrar de una generación a otra? ¿Bienes básicos, instituciones? Rawls no es muy claro sobre esto, pues finalmente dice que no se trata únicamente de ahorro monetario o de capital, sino de instituciones justas, pero English recalca que no es evidente cómo eso puede traspasarse de una generación a otra.

La propuesta de English, entonces, consiste en tomar a las partes en la PO como individuos, en lugar de como cabezas de familia. También, eliminando el requisito del momento presente de entrada como factor para la decisión de los principios de justicia. Al hacer esto obtenemos que, como una cuestión de justicia, los mejor situados en una generación deben ahorrar para los peor situados en la siguiente, así como los mejor situados en la generación siguiente deben proveer para los peor situados en la generación anterior (English 1977, 103). Esto permitiría garantizar el ahorro intergeneracional, sin caer en las asunciones complejas sobre el carácter de las familias. Pero no resuelve todo el problema de la familia que se desprende de la TJ.

Veronique Munoz-Darde (1998) ha hecho un importante aporte sobre la cuestión de la familia en la teoría de Rawls, distinguiendo entre la justicia 'al interior' de la familia y la justicia 'de' la familia. La primera hace referencia a la crítica sobre la familia como parte o no de la estructura básica de la sociedad y sobre si los beneficios y cargas, al interior de ella, son distribuidos de manera justa. La segunda hace referencia a si la existencia de la familia como tal, es justa.

Munoz-Darde afirma que la teoría de Rawls funcionaría mejor si fuera más individualista, siguiendo la estela de English. Pero se hace cargo de cuestiones a las que English no pone atención, como el impacto negativo de la familia sobre la igualdad de oportunidades. Para la autora, Rawls no persevera en sugerir la posibilidad de la abolición de la familia, por alguna de estas causas:

- la familia es tratada como un 'hecho bruto' (o como un hecho inevitable), o

- la necesidad de esta institución es justificada por razones más poderosas que sus efectos injustos.

Munoz-Darde (1998) se inclina por la segunda afirmación y señala que la familia, más allá de ser una institución necesaria para la formación del sentido moral, es necesaria para garantizar que las partes en la PO puedan representarse los intereses de otras generaciones. 
La autora propone dos modificaciones a la teoría de Rawls: que no se considere a las partes en la PO como cabezas de familia y que no se asuma en la PO que las familias se preocupan de alguna manera especial por sus miembros.

\section{La crítica de Okin}

Susan Moller Okin dedica un capítulo de su libro Justice, Gender and the Family (2007) a analizar TJ. Allí identifica varios fallos que darían cuenta de una cierta ceguera por parte de Rawls respecto de los temas de género. Quizás el más sobresaliente de ellos es que Rawls no menciona el sexo entre aquellos aspectos que deberían quedar tras el velo de ignorancia. Asimismo, tampoco considera la dependencia económica de las mujeres respecto de los hombres como parte de la estructura básica. Sin embargo, asume que la familia es justa y que es el espacio donde se debe formar el sentido de la justicia de los futuros ciudadanos, considerando como diferenciadas aquellas virtudes que corresponden a 'la buena hija,' 'el buen hijo', 'la buena esposa', 'el buen esposo'. Su ruptura aquí con el masculino universal que atraviesa todo el texto hace intuir que se trataría de virtudes diferenciadas.

Okin se reconoce a sí misma como rawlsiana y desea rescatar el potencial de las ideas de TJ para el feminismo. Es por ello que señala dos aspectos que urge corregir de la teoría para activar dicho potencial: el primero es considerar el sexo como uno de los aspectos que deberían quedar explícitamente tras el velo de ignorancia; el segundo, que explícitamente se defina que uno de los 'hechos generales de la sociedad humana' de los que las partes tienen conocimiento, sea el que la sociedad está estructurada sobre una jerarquía de géneros. Así, es más probable que la familia sea sujeta a un escrutinio al que Rawls no la somete. Okin $(2007,105)$ lleva este razonamiento al punto de afirmar que "nuestra actual estructura de género es incompatible con el logro de la justicia social". Para Okin, la justicia social en clave feminista solo es posible en una sociedad sin géneros.

\section{La familia y la justicia más allá del marco rawlsiano}

Las posibilidades de una crítica feminista de la familia pueden ir en la dirección radical hacia las que las enfoca Okin (2007), según la cual solo 
con la abolición del género puede lograrse una verdadera justicia feminista, al menos en términos distributivos, pues, abolido el género, se eliminaría la división sexual del trabajo. Esto implica una particular posición sobre las cuestiones de reconocimiento, sobre la que no hay acuerdo en el feminismo y que probablemente tampoco sería aceptada como una doctrina razonable desde el punto de vista de lo expuesto por Rawls en Liberalismo político (1993).

Como sea, creo que la posición feminista sobre la familia supone considerarla parte de la estructura básica, a la altura de "las principales disposiciones económicas y sociales" (Rawls 2005, §2), y no meramente como una asociación voluntaria (Rawls 2001), con lo cual se rompe la idea de su armonía interna y se visibilizan los intereses conflictivos que en ella residen. Hacerla sujeto de la justicia probablemente implique un nivel mayor de intervención que el que una teoría liberal estaría dispuesta a asumir. Pero se trata del costo de una teoría que pretenda hacerse cargo de la porosidad de los límites entre lo público y lo privado. ¿Cuál es el carácter de dicha intervención? Es algo que todavía está por definirse, pero donde inevitablemente la libertad individual entra en conflicto con las pretensiones de justicia. La idea de que lo personal es político no es un ideal fácil de satisfacer.

\section{Bibliografía}

English, J. 1977. Justice between Generations. Philosophical Studies 31(2), 91-104. DOI: https://doi.org/10.1007/BF01857179.

Firestone, S. 1970. The Dialectic of Sex: The Case for Feminist Revolution. New York: Quill. Millett, K. 2000. Sexual Politics. Urbana: University of Illinois Press.

Morgan, R. (ed.) 1970. Sisterhood Is Powerful. New York: Random House.

Munoz-Darde, V. 1998. Rawls, Justice in the Family and Justice of the Family. Philosophical Quarterly 48(192), 335-352. DOI: https://doi.org/10.1111/14679213.00104.

Okin, S.M. 2007. Justice, Gender, and the Family. New York: Basic Books.

Rawls, J. 1993. Political Liberalism. New York: Columbia University Press.

Rawls, J. 2001. Justice as Fairness: A Restatement. Cambridge, MA: Harvard University Press.

Rawls, J. 2005. A Theory of Justice. Original Edition. Cambridge, MA: Harvard University Press. EP 